\title{
Chronische Zystitiden: kausale Therapie mit Chondroitinsulfat
}

- Die Symptome von interstitieller Zystitis (IC) und überaktiver Blase (OAB) haben häufig die gleiche Ursache: eine Schädigung der Glykosaminoglykan-(GAG)-Schicht als Grenze zwischen Urothel und Urin. Die intravesikale Instillation mit 0,2\%igem Chondroitinsulfat (Gepan ${ }^{\circledR}$ instill) versorgt die GAG-Schicht mit ihrem wichtigen Baustein Chondroitinsulfat und behebt den Defekt der Blasenschutzschicht.

Die IC ist eine chronische, nicht erregerbedingte Zystitis unklarer Ursache mit progredientem Verlauf, suprapubischen Schmerz und hoher Miktionsfrequenz. Zusätzlich zur Therapie mit hauptsächlich symptomatisch wirkenden Medikamenten wie trizyklischen Antidepressiva ist die GAG-Ersatztherapie mit Chondroitinsulfat eine bewährte Behandlungsoption der IC. Im Rahmen einer Beobachtungsstudie an 286 Patienten mit unterschiedlichen Zystitisformen, die mit Gepan ${ }^{\circledR}$ instill behandelt worden waren, reduzierte sich die Miktionsfrequenz von 17 auf 11 und die Intensität des Harndrangs halbierte sich.

Die OAB ist charakterisiert durch imperativen Harndrang, der bei etwa einem Viertel der Betroffenen zu einem unkontrollierten Harnverlust, der Dranginkontinenz, führt. Typischerweise haben die Patienten zu- sätzlich eine erhöhte Miktionsfrequenz. Wird der GAG-Defekt geheilt, verbessern sich die klinischen Symptome der OAB deutlich stärker als unter der Behandlung mit Anticholinergika. In einer kontrollierten Studie an 82 Patienten mit OAB besserten sich nach einem Jahr Therapie mit Gepan ${ }^{\circledR}$ instill bei $74 \%$ der Patienten die Symptome. Zwölf Monate nach Ende der Studie zeigten noch $56 \%$ der Patientinnen mit GAG-Ersatz eine Symptomverbesserung, in der Tolterodingruppe $14 \%$.

Nach Informationen von

Pohl-Boskamp, Hohenlockstedt

\section{Erektile Dysfunktion: Schmelztablette für mehr Spontanität}

Levitra $^{\circledR}$ (Vardenafil $\mathrm{HCl}$ ) zur Behandlung der Erektilen Dysfunktion (ED) ist bald auch als $10 \mathrm{mg}$-Schmelztablette erhältlich. Die Europäische Kommission hat kürzlich die Marktzulassung für die neue Formulierung erteilt. Das Besondere an der neuen Pillenart: Die Schmelztablette mit Minzgeschmack löst sich ohne Wassereinnahme sekundenschnell auf der Zunge auf. Die Einnahme ist somit sehr diskret und angenehm. Die Marktausbietung soll ab November 2010 in Europa beginnen. In den USA wurde das Medikament bereits im Juni 2010 zugelassen.

„Wir sind davon überzeugt, dass Männer ED-Medikamente bevorzugen, die auf ihre
Bedürfnisse zugeschnitten und leicht einzunehmen sind. Die neue Levitra Schmelztablette ermöglicht Männern mit ED ihr ED Medikament jederzeit und ohne Wasser einzunehmen. Dies unterstützt die sexuelle Spontanität“, erläutert Dr. Flemming Oernskov, Leiter der Business Unit Women's Healthcare und General Medicine bei Bayer Schering Pharma.

Die zwei multinationalen Phase-III-Studien POTENT I und II haben die neue Vardenafil-Formulierung untersucht. In den Zulassungsstudien wurde die Wirksamkeit und Sicherheit der Schmelztablette mit Placebo verglichen. Studienteilnehmer waren 882
Männer über 18 Jahren, die bereits seit mehr als sechs Monaten Erektionsstörungen hatten. Die zwei Studien wurden mit identischem Studiendesign über einen Behandlungszeitraum von zwölf Wochen durchgeführt. In beiden Analysen erwies sich die Behandlung mit der Vardenafil$10 \mathrm{mg}$-Schmelztablette als statistisch signifikant überlegen gegenüber Placebo, und zwar in allen primären (IIEF-EF, SEP2, SEP 3 ) und sekundären Wirksamkeitskriterien ( $p<0,0001$ für alle Kriterien).

Nach Informationen von Bayer Schering, Berlin

\section{BPS-Therapie: Patienten profitieren von hoher $\alpha_{1 A}$-Selektivität}

— Das Benigne Prostatasyndrom (BPS) ist eine der häufigsten Erkrankungen älterer Männer. Jeder zweite Mann über 60 Jahre und mehr als $80 \%$ der Männer über 80 Jahre leiden an BPS. $\alpha$-Rezeptorenblocker sind dabei die wichtigste Therapiesäule beim BPS. Zum ersten Mal seit fast 15 Jahren steht nun mit dem Wirkstoff Silodosin (Uro$\mathrm{rec}^{\circledR}$ ) eine neue Therapieoption aus dieser Arzneimittelklasse zur Verfügung.

Silodosin ist ein $\alpha$-Blocker mit hoher Selektivität für $\alpha_{1 A}$-Rezeptoren und wirkt so gezielt im Urogenitaltrakt. Der in der Gefäßwand lokalisierte $\alpha_{1 \mathrm{~B}}$-Rezeptor regelt durch Gefäßverengung den Blutdruck. Werden diese $\alpha_{1 \mathrm{~B}}$-Adrenozeptoren blockiert, kommt es zu einem unerwünschten Blutdruckabfall und Orthostasen, was sich gerade bei kardial vorbelasteten Patienten negativ auswirkt. Silodosin hat mit einem $\alpha_{1 \mathrm{~A}} z u \alpha_{1 \mathrm{~B}}$ Bindungsverhältnis von 162:1 die mit Abstand höchste Uroselektivität aller Alphablocker und blockiert so nahezu nur die Rezeptoren, die im Zusammenhang mit den BPS-Beschwerden stehen. Die klinische Wirksamkeit von Silodosin wurde in mehreren placebokontrollierten Studien nachgewiesen. Bei hochsignifikanter Überlegenheit im IPS-Score überzeugte Silodosin vor allem durch eine sehr schnelle und langanhaltende Wirkung. In einer direkten klinischen Vergleichsstudie versus Tamsulosin war Silodosin in der Verbesserung des sehr unangenehmen BPS-Symptomkomplexes aus Nykturie, Miktionsfrequenz und Restharngefühl signifikant überlegen. Silodosin bietet aufgrund seiner hohen Uroselektivität für $\alpha_{1 A}$-Rezeptoren ein ausgezeichnetes kardiovaskuläres Sicherheitsprofil, auch in Komedikation mit PDE5-Hemmern. Dabei ergaben sich keine klinisch relevanten pharmakodynamischen Wechselwirkungen.

Nach Informationen von

Merckle-Recordati, Ulm 